\title{
Isolamento de Candida spp. no mamilo de lactantes do Banco de Leite Humano da Universidade Federal do Ceará e teste de susceptibilidade a antifúngicos
}

\author{
Candida ssp. isolation in the breastfeeding mothers' nipples from the human milk bank at the Universidade \\ Federal do Ceará and susceptibilities to the antifungal agents tests
}

Everardo Albuquerque Menezes ${ }^{1 ;}$ Anne Cristina Pinheiro Guerra²; Rita de Cássia Barros Rodrigues; Maria Marly Lopes Vieira Peixoto4; Liliane Santiago Lima²; Francisco Afrânio Cunha ${ }^{5}$

unitermos
Candidiase mamária
Aleitamento materno
Fator de virulência
Mastite
Candida albicans

\section{resumo}

A candidíase, principal infecção fúngica oportunista do ser humano, é provocada por leveduras do gênero Candida que fazem parte da microbiota endógena do corpo humano. $\mathrm{O}$ objetivo deste trabalho foi estudar as espécies de Candida envolvidas em processos inflamatórios da mama em mães que estavam amamentando no Banco de Leite Humano da Maternidade-Escola Assis Chateaubriand da Universidade Federal do Ceará (UFC), verificando as atividades enzimáticas e as suscetibilidades aos principais antifúngicos usados na candidíase. As amostras foram colhidas de fissuras no mamilo, cultivadas em ágar Sabouraud a $37^{\circ} \mathrm{C}$ por 72 horas e identificadas por testes micológicos. Foram realizadas também provas da proteinase, fosfolipase e hialuronidase e testes de susceptibilidade a anfotericina B e fluconazol. Das 29 amostras, 13 (44,8\%) apresentaram crescimento; 11 (84,6\%) foram Candida albicans; uma $(7,7 \%)$, C. stellatoidea; e uma $(7,7 \%)$, C. guilliermondii. As atividades enzimáticas de C. albicans foram: $100 \%$ das cepas produziram fosfolipase; $64 \%$, hialuronidase; e nenhuma produziu proteinase. As leveduras examinadas mostraram-se susceptíveis aos antifúngicos testados com concentração inibitória mínima (CIM) de: anfotericina B 0,125 a 0,5 $\mathrm{g} / \mathrm{ml}$ e fluconazol 2 a $4 \mu \mathrm{g} / \mathrm{ml}$. Todas as cepas de C. albicans isoladas do mamilo de mães que estavam amamentando mostraram a enzima fosfolipase, que pode ser um importante fator de virulência, e apresentaram uma excelente susceptibilidade aos antifúngicos testados. abstract

Candidiasis, human being's main opportunist fungal infection, is caused by Candida, a sort of yeasts which are part of endogenous microflora of the human body. The purpose of this research was to study the Candida species involved in the inflammatory processes of the breastfeeding mothers' nipples from the human milk bank on Assis Chateubriand maternity-school at the Universidade Federal do Ceará, Brazil. It was verified the enzymatic activities and the susceptibilities to the main used antifungal agents in candidiasis. The samples were collected from the wounds in the nipples and grown in Sabouraud agar at $37^{\circ} \mathrm{C}$ for 72 hours. They were identified by mycology tests. Proteinasis, phospholipase and hialuronidase tests were also made. Amphotericin $B$ and fluconazole susceptibility tests, too. From the 29 samples, 13 (44. 8\%) of the total presented growth; 11 (84.6\%) of them were Candida albicans; 01 (7.7\%) of them was C. stellatoidea and 01 (7.7\%) of them was C. guilliermondii. The C. albicans enzymatic activities were: $100 \%$ of strains produced phospholipase; $64 \%$ produced hialuronidase and none of the strains produced proteolytic activity. The tested yeasts were susceptive to the antifungal agents tested with minimum inhibitory concentration $-0.125-0.5 \square \mathrm{g} / \mathrm{ml}$ for amphotericin $B$ and $2-4 \square \mathrm{g} / \mathrm{ml}$ for fluconazol. All the C. albicans strains isolated from the breastfeeding mothers' nipples showed the phospholipase enzyme which can be an important virulence factor. They presented excellent susceptibilities to the antifungal agents tested. key words

Nipple candidiasis

Breastfeeding mothers

Virulence factor

Mastitis

Candida albicans

1. Professor-doutor do Departamento de Análises Clínicas e Toxicológicas (DACT) da Universidade Federal do Ceará (UFC).

2. Aluna do curso de Farmácia da Faculdade de Farmácia, Odontologia e Enfermagem (FFOE) da UFC.

3. Enfermeira do Banco de Leite Humano da UFC.

4. Farmacêutica-bioquímica do Banco de Leite Humano da UFC.

5. Farmacêutico-bioquímico do DACT/FFOE/UFC.

Financiado pela Fundação Cearense de Apoio ao Desenvolvimento Científico e Tecnológico (FUNCAP). 


\section{Introdução}

A infecção mamilar é um problema que está crescendo na lactação, sendo que a causada por fungos (candidíase mamilar) ainda é pouco estudada e elucidada. A Candida albicans, um fungo comensal encontrado freqüentemente na vagina e no trato gastrointestinal de seres humanos, tem sido responsabilizada por infecção superficial e localizada das mamas em mulheres lactantes, apresentando fissuras e dor, sendo característica da infecção pela levedura e podendo ser a principal causa de abandono prematuro da lactação(10).

A candidíase, principal infecção fúngica oportunista do ser humano, é provocada por leveduras do gênero Candida, que fazem parte da microbiota endógena do corpo humano. Essa infecção expressa muito bem a variedade de relações que ocorrem entre o hospedeiro e a microbiota autóctone, podendo ir do comensalismo à doença sistêmica fatal ${ }^{(8)}$. Candida albicans é a principal espécie do gênero associada à candidíase, mas outras também são relatadas, como por exemplo: Candida tropicalis, Candida glabrata, Candida krusei, Candida parapsilosis, Candida kefir e Candida guilliermondii.

As formas clínicas podem ser: mucosa, cutânea e sistêmica. Na candidíase mucosa, os tecidos mais atingidos são os da boca e da vagina; na cutânea, as áreas intertriginosas da pele das mãos, as virilhas e as axilas são comprometidas; na sistêmica, a infecção pode atingir diversos órgãos, causando candidíase pulmonar, endocardite, nefrite e fungemia ${ }^{(1-3)}$. Dependendo da localização, a candidíase pode se manifestar de diferentes formas. Os achados clínicos na candidíase oral são bastante variáveis, podendo ser observados desde quadros localizados até formas extensas.

As lesões, conhecidas no Brasil pela denominação popular de sapinho, são mais comuns em recém-nascidos que ainda não colonizaram sua orofaringe, que por isso possui um pH baixo, condição que facilita a colonização da cavidade oral. Clinicamente inicia-se por pequenos pontos esbranquiçados na mucosa que rapidamente se tornam confluentes, para formar pseudomembranas de coloração esbranquiçada, aderidas à mucosa sobre um fundo eritematoso, que pode ser visto quando são removidas. As localizações mais comuns na cavidade oral são as mucosas que revestem bochechas, ponta da língua e palato mole. Entretanto, numa forma mais extensa, pode ser observada uma invasão maciça, com extenso comprometimento da cavidade oral, dificultando muitas vezes a deglutição, sendo esta entidade mais observada em recém-nascidos de mães portadoras de candidíase vulvovaginal( ${ }^{(9)}$. Atualmente a $C$. albicans tem sido responsabilizada por candidíase mamilar em mulheres lactantes, sendo a dor e o ardor no mamilo de origem desconhecida as principais queixas de infecção por essa levedura ${ }^{(10)}$.

As décadas de 1980 e 1990 caracterizaram-se por um aumento do número de mães decididas a amamentar. Apesar disso, a prática indica que um número significativo destas param de amamentar logo em seguida. Entre as várias razões para essa interrupção, pode ser citada a fissura da região mamilo-aréola, com dor e vermelhidão mamilar, conseqüência da Candida albicans ${ }^{(14)}$. É importante que os profissionais de saúde, as mulheres lactantes e os demais envolvidos na educação do processo de aleitamento materno trabalhem para compreender melhor a candidíase mamilar ${ }^{(10)}$.

Apesar do aumento no número de antifúngicos comercialmente disponíveis nos últimos anos, eles ainda encontram-se em desvantagem, quando comparados às drogas antibacterianas. A resistência aos antifúngicos tem representado um grande desafio para a clínica. Frente às dificuldades observadas no tratamento de micoses em alguns grupos de pacientes, recomenda-se, sempre que possível, o isolamento do agente responsável pela infecção e a determinação da concentração inibitória mínima (CIM) das drogas passíveis de utilização(4).

Em função do grande número de lactantes atendidas no Banco de Leite Humano da Maternidade-Escola Assis Chateaubriand da Universidade Federal do Ceará (BLH/ MEAC/UFC), com queixas de fissura na base do mamilo, despigmentação mamilar e aureolar e ardor (ou queimação) ao amamentar, como também do crescente interesse pelos mecanismos de virulência de Candida, surgiu o nosso interesse em identificar as espécies envolvidas em processos inflamatórios da mama em pacientes atendidas no BLH/MEAC/UFC. Além de verificar os mecanismos de virulência das leveduras, investigamos o grau de resistência destas aos principais antifúngicos usados nas terapias das candidíases.

\section{Material e método}

As amostras foram colhidas em pacientes atendidas no BLH/MEAC/UFC. A população compreendeu mulheres que estavam amamentando e apresentavam quadro de mastite, ou seja, com fissuras na base do mamilo com eritema, sugestiva de candidíase mamilar, e que não estavam sendo tratadas. As amostras foram colhidas das fissuras da 
região mamilo-aréola (Figura 1), com swabs esterilizados e transportados, em meio salino, para o Laboratório de Micologia do Departamento de Análises Clínicas e Toxicológicas (DACT) da UFC. As amostras, colhidas no período de março de 2001 a janeiro de 2002, foram semeadas em ágar Sabouraud $4 \%$ de glicose com cloranfenicol e incubadas a $37^{\circ} \mathrm{C}$ por três a cinco dias. As colônias foram identificadas segundo os métodos micológicos tradicionais ${ }^{(7)}$ : zimograma, que consiste na diferenciação das leveduras, utilizando a habilidade de cada espécie em fermentar açúcares; teste do tubo germinativo, que observa a formação de um tubo em espécies de leveduras do gênero Candida; teste dos clamidósporos em ágar fubá com Tween 80, que avalia a formação de estruturas características de cada espécie, quando há crescimento em meio com poucos nutrientes ${ }^{(8)}$.

Nas amostras identificadas como C. albicans, foram realizados os testes de fatores de virulência: fosfolipase, proteinase e hialuronidase ${ }^{(18,21)}$. A presença de enzimas foi observada pela formação de uma zona opaca ao redor da colônia da levedura, e a atividade enzimática (PZ) foi medida, dividindo-se o diâmetro da colônia pelo diâmetro da colônia mais a zona de precipitação. A PZ foi codificada com dígitos, para as cepas não-produtoras dessa enzima $(P Z=1)$, para atividade enzimática média $(1>P Z \geq 0,64)$ e para atividade enzimática elevada $(P Z \leq 0,63)^{(17)}$.

Por fim, foram realizados os testes de susceptibilidade aos antifúngicos: anfotericina B (Bristol-Myers-Squibb) e fluconazol (Pfizer), segundo a metodologia do National Commitee for Clinical Laboratory Standards (NCCLS) (protocolo $\left.\mathrm{n}^{\circ} \mathrm{M} 27 \mathrm{~A}\right)^{(16)}$, de macrodiluição em caldo ${ }^{(16)}$. Foi determinada a CIM. As concentrações de anfotericina B utilizadas foram de $0,125 \mu \mathrm{g} / \mathrm{ml}$ a $32 \mu \mathrm{g} / \mathrm{ml}$. Para o fluconazol foram utilizadas concentrações de $1 \mu \mathrm{g} / \mathrm{ml}$ a $128 \mu \mathrm{g} / \mathrm{ml}$. Foi considerada CIM a menor concentração do antifúngico que impediu crescimento visível da levedura.

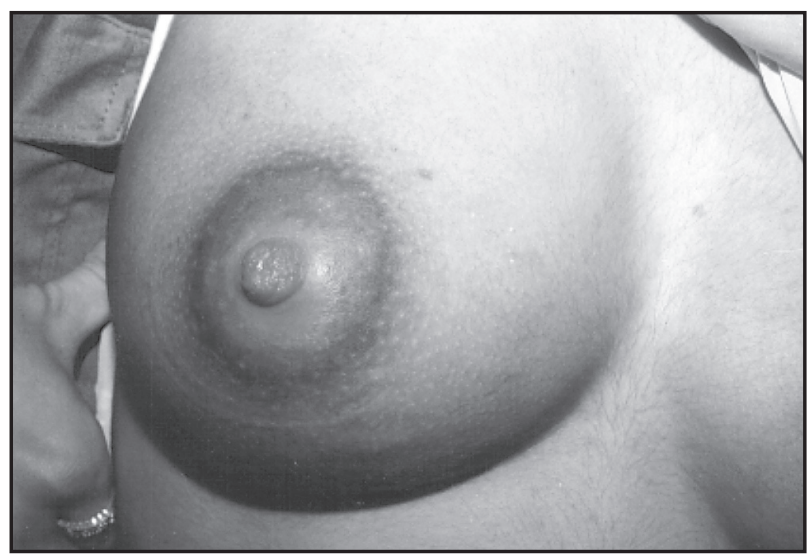

Figura 1 - Fissura na base do mamilo de uma lactante do BLH/MEAC/UFC
Cepas que apresentam $\mathrm{CIM} \leq 8 \mu \mathrm{g} / \mathrm{ml}$ para o fluconazol são consideradas sensíveis; CIM entre 16 e $32 \mu \mathrm{g} / \mathrm{ml}$, intermediárias; e cepas com $\mathrm{CIM} \geq 64 \mu \mathrm{g} / \mathrm{ml}$, resistentes ${ }^{(19)}$. Cepas que apresentam $\mathrm{CIM} \leq 1 \mu \mathrm{g} / \mathrm{ml}$ para a anfotericina B são consideradas sensíveis; CIM entre 2 e $4 \mu \mathrm{g} / \mathrm{ml}$, intermediárias; $\mathrm{CIM} \geq 4 \mu \mathrm{g} / \mathrm{ml}$, resistentes ${ }^{(16)}$. A CIM dos azólicos é definida como a menor concentração da droga que resulta em decréscimo da turbidez comparado com um controle ${ }^{(16)}$. Como controle do teste, as amostras cresciam em meio de cultura livre do antifúngico.

\section{Resultados}

Das 29 amostras, 13 (44,8\%) apresentaram crescimento de leveduras do gênero Candida. Das amostras positivas, 11 $(84,6 \%)$ foram identificadas como Candida albicans, uma $(7,7 \%)$ como Candida stellatoidea e uma $(7,7 \%)$ como Candida guilliermondii (Figura 2). Foi verificado que a Candida albicans foi a mais prevalente.

A Tabela 1 mostra a PZ das amostras de C. albicans, observando-se que todas as cepas estudadas não apresentaram ação proteolítica, isto é, apresentaram $P Z=1$. Em relação à ação fosfolipásica foi verficado que $45,4 \%$ apresentaram atividade enzimática moderada, entre 0,64 e 0,99, e 54,6\%, uma elevada atividade enzimática, PZ menor do que 0,63. A hialuronidase apresentou uma grande atividade enzimática em 64\% das Candida albicans, com PZ menor do que 0,63, sendo observado que $36 \%$ das amostras não apresentaram ação da enzima hialuronidase (Figura 3).

A Tabela 2 mostra o perfil da CIM das amostras de $C$. albicans em relação aos antifúngicos testados: anfotericina $B$ e fluconazol. A primeira teve uma CIM que variou de 0,125 a $0,5 \mu \mathrm{g} / \mathrm{ml}$ e a segunda, de 2 a $4 \mu \mathrm{g} / \mathrm{ml}$. Foi observado que todas as cepas testadas foram sensíveis.

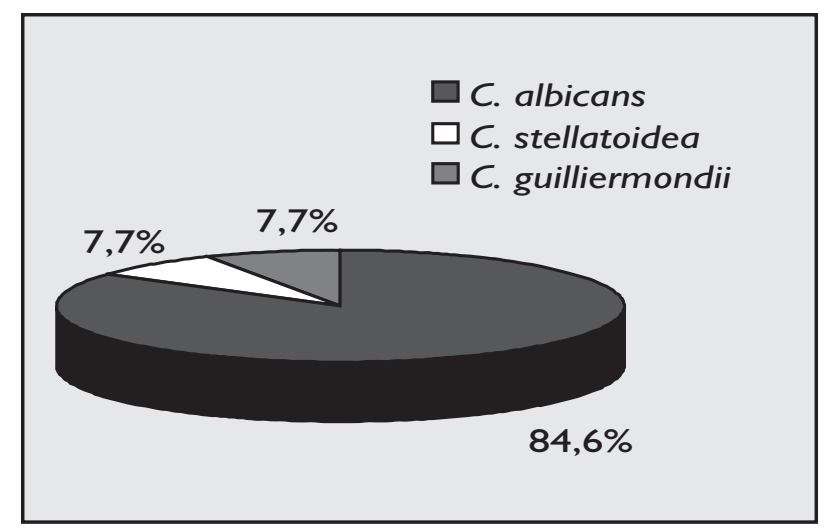

Figura 2 - Distribuição das cepas de Candida isoladas de lactantes com mastite no BLH/MEAC/UFC 


$\begin{array}{cccc}\text { Tabela } 1 & \text { Fosfolipase } & \text { Proteinase } & \text { Hialuronidase } \\ \text { Amostra } & 0,59 & 1 & 0,52 \\ 1 & 0,57 & 1 & 0,56 \\ 2 & 0,64 & 1 & 0,48 \\ 3 & 0,69 & 1 & 0,47 \\ 4 & 0,7 & 1 & 1 \\ 5 & 0,66 & 1 & 0,39 \\ 6 & 0,92 & 1 & 0,6 \\ 7 & 0,62 & 1 & 0,58 \\ 8 & 0,59 & 1 & 1 \\ 9 & 0,43 & 1 & 1 \\ 10 & 0,43 & 1 & 1 \\ 11 & & & \end{array}$

PZ = atividade enzimática.

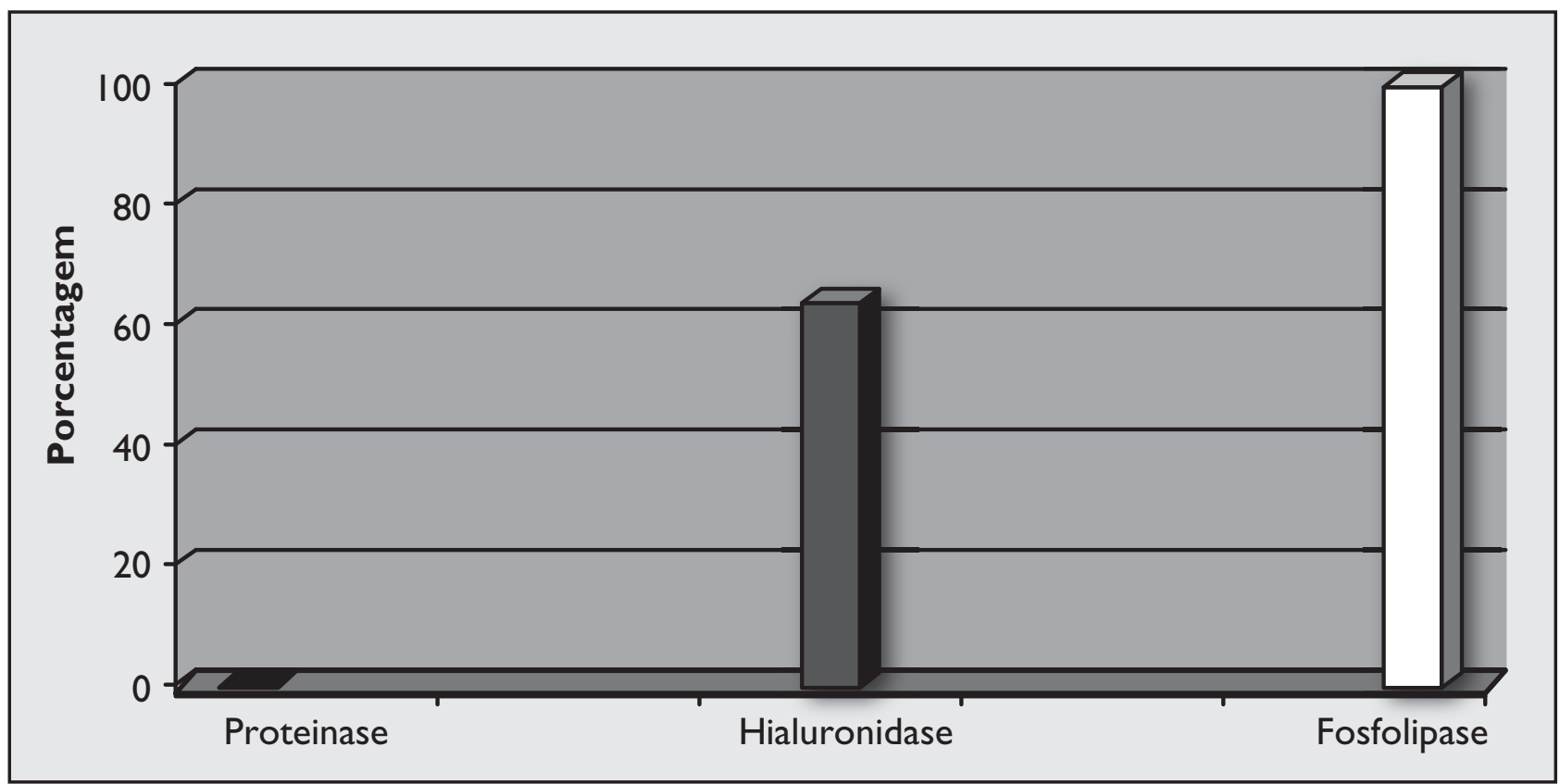

Figura 3 - Atividades enzimáticas de Candida albicans isoladas de lactantes com mastite no BLH/MEAC/UFC

A Figura 4 mostra a CIM de anfotericina B das cepas de Candida albicans isoladas de mastites. Os resultados foram cinco cepas com CIMs equivalentes a $0,5 \mu \mathrm{g} / \mathrm{ml}$, quatro com CIMs de $0,25 \mu \mathrm{g} / \mathrm{ml}$ e duas cepas com CIMs de $0,125 \mu \mathrm{g} / \mathrm{ml}$. A Figura 5 mostra a CIM de fluconazol das cepas de Candida albicans isoladas neste estudo. Os resultados mostram que sete cepas tiveram CIMs equivalentes a $4 \mu \mathrm{g} / \mathrm{ml}$ e quatro, CIMs de $2 \mu \mathrm{g} / \mathrm{ml}$.

\section{Discussão}

Em nosso estudo, usamos como critérios de inclusão as infecções mamilares de mulheres que estavam amamentando e freqüentavam o BLH/MEAC/UFC. Elas apresentavam fissuras na base do mamilo com eritema, o diagnóstico de mastite foi clínico e as mulheres não estavam fazendo uso de medicamentos. A coleta foi 
realizada da fissura da região mamilo-aréola (Figura 1), e foram isoladas leveduras, sendo a espécie Candida albicans a mais isolada, o que está de acordo com outras publicações. Jarvis ${ }^{(11)}$, em um estudo epidemiológico realizado de 1980 a 1990, analisando 180 diferentes

\begin{tabular}{ccc} 
Tabela 2 & BLH/MEAC/UFC & \\
\hline Amostra & Anfotericina B & Fluconazol \\
1 & 0,5 & 4 \\
2 & 0,25 & 4 \\
3 & 0,25 & 2 \\
4 & 0,5 & 4 \\
5 & 0,125 & 2 \\
6 & 0,25 & 2 \\
7 & 0,25 & 4 \\
8 & 0,5 & 4 \\
9 & 0,125 & 4 \\
10 & 0,5 & 4 \\
11 & 0,5 & 2 \\
\hline
\end{tabular}

hospitais, verificou que $72,1 \%$ das infecções fúngicas eram devidas às espécies de Candida.

Dor durante a amamentação, com ausência de sinais sistêmicos, pode ser um indicativo de infecção por $C$. albicans. O seio pode aparecer com eritema ou fissurado. Tanguay et al. (22), estudando 105 mulheres que amamentavam, observaram que $27(25,7 \%)$ apresentavam diagnóstico para candidíase mamária.

Num estudo realizado com 61 mulheres com dor no mamilo e que estavam amamentando, 64 mulheres sem dor no mamilo e que estavam amamentando, e $31 \mathrm{mu}$ Iheres que não tinham dor no mamilo e que não estavam amamentando, como caso controle, foi observado que, em $19 \%$ das coletas daquelas que apresentavam dor no mamilo e estavam amamentando, aumentou a quantidade de $C$. albicans, sendo significativamente maior que no grupo controle $^{(1)}$. Em nosso estudo, o índice encontrado foi de $44,8 \%$, o que demonstra uma elevação nos índices dessa doença (Figuras 1 e 2).

A candidíase mamária pode estar associada a vários fatores, como lesão no mamilo no início da lactação, mastite, uso de antibióticos pós-parto e candidíase vulvovaginal(22). A levedura mais comumente isolada em nosso estudo foi a C. albicans. Quando este patógeno infecta mães lactantes, $\mathrm{o}$ ato de amamentar se torna extremamente doloroso, o que pode levar a um desmame prematuro(6).

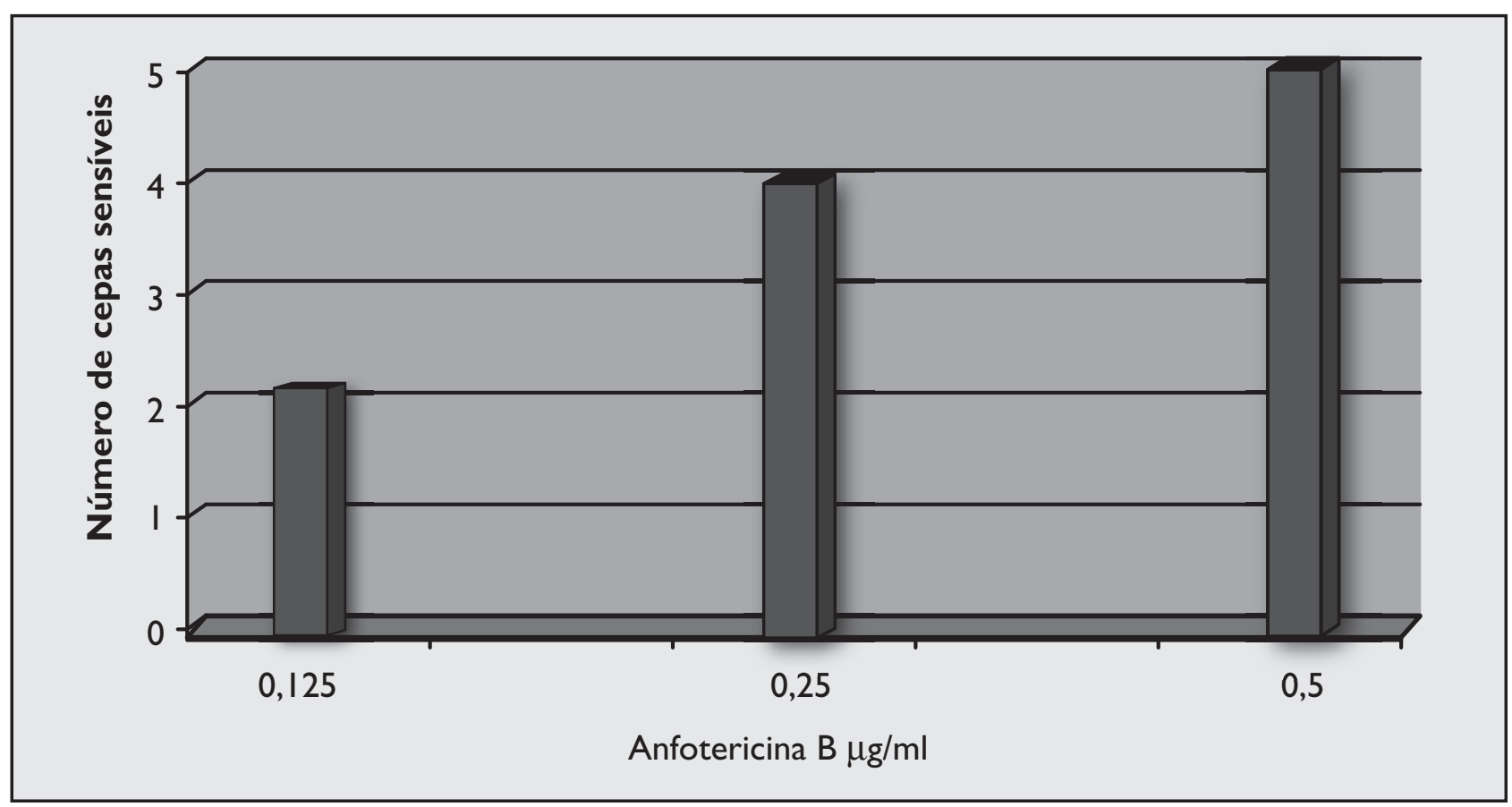

Figura 4 - Número de cepas de Candida albicans sensíveis à CIM de anfotericina B, isoladas de lactantes com mastite no BLH/MEAC/UFC 


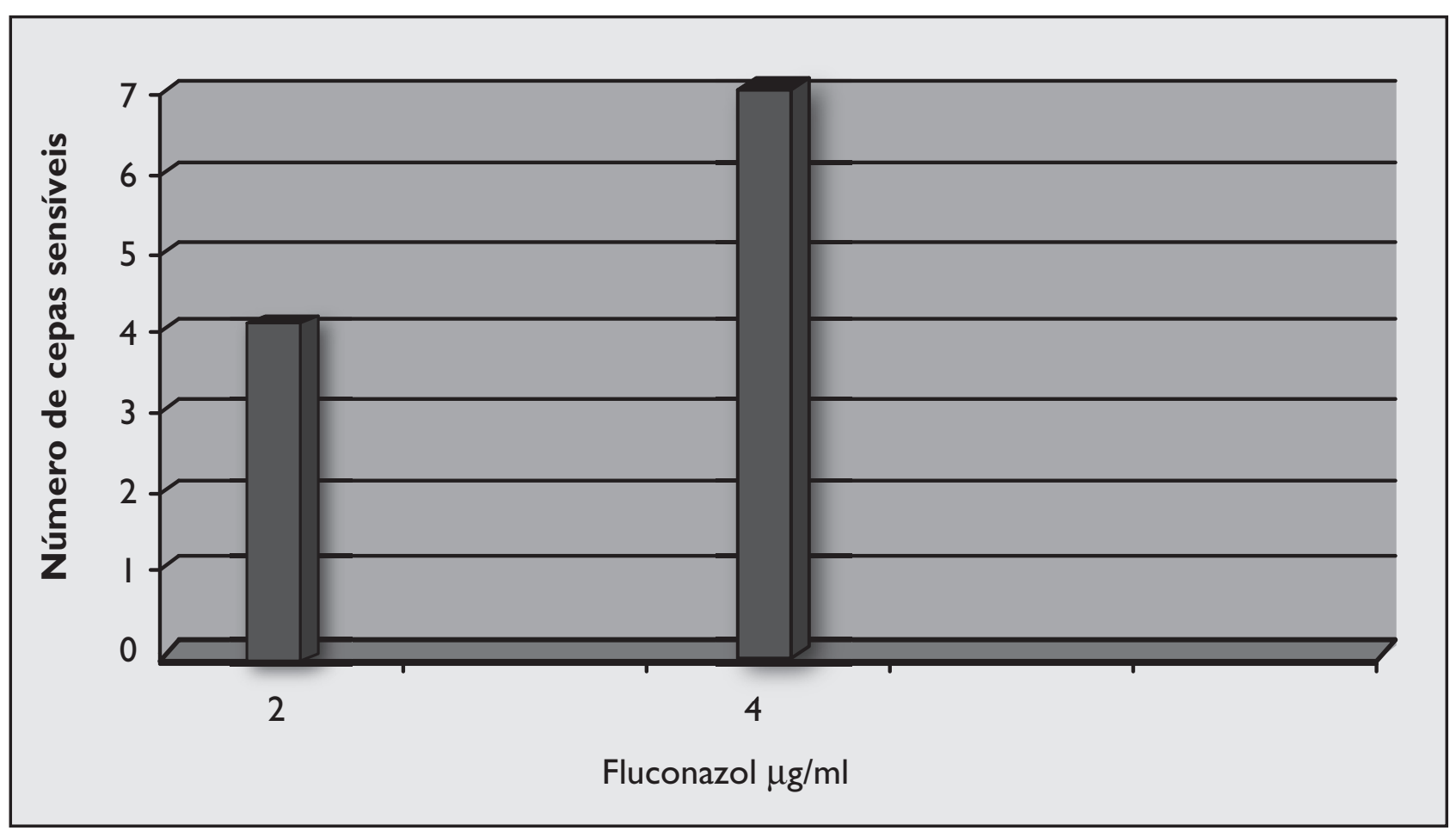

Figura 5 - Número de cepas de Candida albicans sensíveis à CIM de fluconazol B, isoladas de lactantes com mastite no BLH/MEAC/UFC

Nesse estudo foram realizados os testes de fatores de virulência, ou seja, exoenzimas secretadas pela levedura com a finalidade de danificar as células do hospedeiro. Entre as enzimas mais importantes secretadas pela C. albicans temos a fosfolipase, que degrada fosfolipídios da membrana das células; a proteinase, que pode degradar anticorpos; e a hialuronidase, que degrada o ácido hialurônico presente entre as células.

Esse repertório enzimático teria a finalidade de facilitar a entrada e a instalação da infecção por parte da $C$. albicans. Para que a enzima secretada seja importante no mecanismo de patogenicidade é necessário que esta esteja em quantidade apreciável. Na Tabela 1 podemos verificar a PZ. Como podemos observar, ocorreu elevada $\mathrm{PZ}$ em relação à fosfolipase e à hialuronidase na maioria das cepas isoladas, com PZ variando de 0,43 a 0,92 para a primeira e 0,39 a 1 para a segunda. Quando a PZ encontrada vale 1, não existe atividade enzimática, fato observado em relação à proteinase ${ }^{(17)}$. Ribeiro ${ }^{(19)}$, estudando os fatores de virulência de C. albicans de 120 amostras de secreção vaginal, identificou $47,5 \%$ de $C$. albicans, todas produtoras de fosfolipases e proteinases. Candido et al. ${ }^{(7)}$ observaram que mais de $83,3 \%$ das cepas isoladas da cavidade bucal de pacientes com candidíase apresentaram forte atividade fosfolipidolítica. Oliveira et al. ${ }^{(17)}$, estudando amostras de C. albicans isoladas da mucosa bucal de pacientes com câncer, verificaram que as amostras apresentavam forte atividade de fosfolipase e proteinase. A virulência de $C$. albicans parece ser não um simples fator, mas sim um conjunto de fatores. Evidências de associações de fatores têm sido recentemente descritas $^{(23)}$. Em nosso estudo, todas as cepas de C. albicans produziram tubo germinativo que, segundo a literatura, é fator importante na colonização e na infecção. É possível que a alta atividade fosfolipidolítica encontrada e a produção de tubo germinativo, bem como a produção de hialuronidase, possam facilitar a penetração na mucosa e o aparecimento característico dos sintomas, como a dor e o rubor no mamilo(23).

Em nosso estudo foram realizados testes de susceptibilidade a dois antifúngicos usados comumente na clínica médica: a anfotericina $\mathrm{B}$ e o fluconazol. O último é menos tóxico e tão eficaz quanto o primeiro em pacientes nãoimunocomprometidos. Porém, existe uma tendência em se evitar o uso de fluconazol no tratamento de candidemias causadas por Candida não-albicans(15). Todas as cepas estudadas foram sensíveis à anfotericina $\mathrm{B}$ e ao fluconazol.

Em estudo realizado por Brent ${ }^{(6)}$, o tratamento mais comum da candidíase mamária foi nistatina creme para a mãe e nistatina oral para o bebê. Segundo Bodley et al. ${ }^{(5)}$, a candidíase mamária pode apresentar uma refratariedade ao tratamento com nistatina creme, sendo necessário o uso 
de fluconazol oral. Nesse caso, a amamentação deverá ser interrompida, pois o fluconazol não deve ser administrado a mulheres lactantes.

A identificação dos patógenos causadores da candidíase mamária e de seus fatores de virulência é importante para se compreender a patologia e usar mecanismos de tratamento, evitando que as mães abandonem ou encurtem o período de amamentação.

\section{Agradecimentos}

Aos funcionários do BLH/MEAC/UFC.

\section{Referências}

I. AMIR, L. H et al. Candida albicans: is it associated wit niplle pain in lactating women? Gynecol Obstet Invest, v. 4I, n. I p. 30-4, 1996.

2.AMIR, L. H. Candida and the lactating breath: prediposing factors, J Hum Lact, v. 7, n. 4, p. 177-81, 1991.

3. AMIR, L. H.; PAKULA, S. Nipple pain, mastalgia and candidiasis in the lactating breast. Aus NZJ Obstet Gynaecol, v. 31 , n. 4, p. 378-80, 1991.

4. BATISTA, J. M.; BIRMAN, E. G.; CURY, A. E. Susceptibilidade a antifúngicos de cepas de Candida albicans isoladas de pacientes com estomatite protética. Rev Odontol Univ São Paulo, v. 13, n. 4, p. 343-8, 1999.

5. BODLEY,V.; POWERS, D. Long-term treatment of a breastfeeding mother with fluconazol - resolved nipple pain caused by yeast: a case study. J Hum Lact, v. 13, n. 4, p. 307- II, 1997.

6. BRENT N. B.Thrush in the breastfeeding dyad: results of a survey on diagnosis and treatment. Clin Pediatr (Phila), v. 40, n. 9 , p. 503-6, 200।.

7. CÂNDIDO, R. C.; AZEVEDO, R. V. P.; KOMESU, M. C. Enzimotipagem de espécies do gênero Candida isoladas da cavidade bucal. Rev Soc Bras Med Trop, v. 33, n. 5, p. 437 42, 2000.

8. FISHER, F.; COOK, N. B. Leveduras e organismos leveduriformes. In: FISHER, F.; COOK, N. B. Micologia: fundamentos e diagnóstico. Rio de Janeiro: Revinter, 200 I, p. 193-226.

9. GROHMANN, P. H.; FRAGA, C. F.A; ABE, A. T. Aids: uma revisão sobre aspectos epidêmicos, imunitários e profiláticos. Folha Médica (Br), v. 95, n. 3, p. 73-8, 1993.

10. HEININ, M. J.; FRANCIS, J.; PAPPAGIANIS, D. Mammary candidosis in lactating womem. J Hum Lact, v. 15, n. 4, p. $281-8$

1 I. JARVIS, R. W. Epidemiology of nosocomial fungal infections, with emphasis on candida species. Clin Infec Dis, v. 20, p. 1526-30, 1995.

12. JOHNSTONE, H. A.; MARCINAK, J. F. Candidiasis in the breastfeeding and infant. J Obstet Gynecol Neonatal Nurs, v. 19, n. 2, p. 17|-3, 1990.

13. KLEIN, R. S. et al. Oral candidiasis in high-risk patients as the initial manifestation of the acquired immunodeficiency syndrome. New Engl J Med, v. 31 I , p. 354-7, 1994.

14. MACDONALD, H. Candida: the hidden deterrent to breastfeeding. Can Nurse, v. 91, n. 9, p. 27-30, 1995.

15. MARRA, A.; CAMARGO, L. F. A. Fluconazol ou anfotericina $B$ no tratamento de candidemias em pacientes internandos na UTI. Rev Assoc Med Bras, v. 48, n. 2, p. I07, 2002.

16. NATIONAL COMMITTEE FOR CLINICAL LABORATORY STANDARDS. Reference method for broth diluition antifungal susceptibility testing of yeast. Approved method M27-A. Villanova, Pennsylvania, National Committee for Clinical Laboratory Standards, 1997.

17. OLIVEIRA, E. E. et al. Toxinas killer e produção de enzimas por Candida albicans isoladas da mucosa bucal de pacientes com câncer. Rev Soc Bras Med Tropic, v. 3 I, n. 6, p. 523-7, 1998.

18. PRICE, M. F.; WILKINSON, M. D; GENTLE, L. O. Plate method for detection of phospholipase activity in Candida albicans. Sabouraudia, v. 20, p. 7-14, 1983.

19. RIBEIRO, E. L. Aspectos biológicos das leveduras do gênero Candida isoladas de candidíase vaginal. Rev Soc Bras Med Trop, v. 3I, n. 6, p. 595, 1997.

20. RODRIGUES-TUDELA, J. L. et al. Correlation of in vitro susceptibility tests result with clinical response: a study of azole therapy in AIDS patients. I Antimicr Chem, v. 35, p. 793-804, 1995.

2।. RUCHEL, J:; TEGELER, R.;TROST, M.A. Comparison of secretory proteinases from differents strains of Candida albicans. Sabouraudia, v. 20, p. 233-44, 1982.

22. TANGUAY, K. E.; MCBEAN, M. R.; JAIN, E. Nipple candidiasis among breastfeeding mothers. Case-control study of predisposing factors. Can Fam Physician, v. 40, p. I407-13, 1994.

23. VIDOTTO,V. et al. Correaltion between germ tube production, phopholipase activity and serotype distribuition in Candida albicans. Rev Iberoam Micol, v. 16, p. 208-10, 1999. 